\title{
Molecular Iodine Clock
}

\author{
Jun Ye,* Long Sheng Ma, and John L. Hall \\ JILA, National Institute of Standards and Technology and University of Colorado, Department of Physics, University of Colorado, \\ Boulder, Colorado 80309-0440
}

(Received 15 June 2001; published 13 December 2001)

\begin{abstract}
We demonstrate a simple optical clock based on an optical transition of iodine molecules, providing a frequency stability superior to most rf sources. Combined with a femtosecond-laser-based optical comb to provide the phase coherent clock mechanism linking the optical and microwave spectra, we derive an rf clock signal of comparable stability over an extended period. Measurements suggest the stability $\left(5 \times 10^{-14}\right.$ at $\left.1 \mathrm{~s}\right)$ of the $\mathrm{cw}$ laser locked on the iodine transition is transferred to every comb component throughout the optical octave bandwidth (from 532 to $1064 \mathrm{~nm}$ ) with a precision of $3.5 \times$ $10^{-15}$. Characterization of the performance of the optical clock shows (in-)stability below $3 \times 10^{-13}$ at $1 \mathrm{~s}$ (currently limited by the microwave sources), and $4.6 \times 10^{-13}$ over one year.
\end{abstract}

DOI: 10.1103/PhysRevLett.87.270801

PACS numbers: 06.20.Fn, 06.30.-k, 42.62.Eh

The recent revolution in physical science brought about by the beautiful merger of $\mathrm{cw}$ based ultraprecision laser work and ultrafast lasers and associated nonlinear optics has enabled profound progress in both areas. Optical frequency measurements have been reduced to a simple task even while the highest level of measurement precision has been achieved [1-5]. Control of the carrier-envelope phase [6] and realization of an optical atomic clock [7] are now possible. Pulse trains from independent mode-locked lasers have been synchronized below 5 fs and their carrier waves phase locked, leading to coherent pulse synthesis $[8,9]$. Arbitrary and yet phase coherent synthesis of optical spectra, either in terms of selecting desired discrete components in the frequency spectrum or by way of specifying preferred pulse shape and duration in the time domain, now appears possible.

To complement the rapid development of high performance optical frequency standards, it is important to establish an optical comb with phase coherence among its individual components exceeding the standards level. With this capability, we will be able to transfer the stability of a single optical oscillator to the entire comb set over its vast bandwidth, and also derive clock signals in the microwave/rf domain without any stability compromise. Optical standards based on single ions and cold atoms promising potential stability around $1 \times 10^{-16}$ at $1 \mathrm{~s}[10]$ and accuracy at $1 \times 10^{-18}$ [11] may very well become future national standards, but require elaborate system designs. On the other hand, excellent candidates in cell-based optical frequency standards do exist, such as the one presented in this Letter, that would offer compact, simple, and less expensive system configurations at the cost of performance degradation by one or two decades. Along with optical combs, a competent laboratory would be able to realize a network of microwave and optical frequencies at a level of stability and reproducibility that surpasses the properties of basically all normal commercially available frequency sources. Easy access to the optical standards would greatly facilitate application of frequency metrology both to precision experiments for fundamental physics and to practical devices.

To reach that goal, it is important to understand and implement an optimized control scheme of the optical comb which would avoid the limitation in phase coherence between the two ends of comb spectrum. In our previous work, an entangled control scheme could achieve only frequency locking across the comb spectrum, with residual frequency noise exceeding $100 \mathrm{~Hz}$ at $1 \mathrm{~s}$ [12]. In this Letter we demonstrate that orthogonal control of the optical comb can lead to $\mathrm{Hz}$ level $\left(\leq 3.5 \times 10^{-15}\right)$ phase-tracking stability across the entire optical octave. Furthermore, the orthogonalization procedure permits independent control of both degrees of freedom associated with the optical comb, leading to a clock work mechanism using only one comb parameter. Recent work [7] uses a two-parameter control to transfer the stability of a cold ion based optical standard to the comb lines at the $3 \times 10^{-16}$ level. Clearly, with a mature technical solution to the "gearbox problem" at hand, all future progress in optical domain and rf domain standards can be utilized in both spectra.

The octave-spanning optical comb and the associated control scheme are shown in Fig. 1. A Kerr-lens modelocked (KLM) femtosecond (fs) (using intracavity prismdispersion compensation) laser [13] generates a repetitive ( $\sim 10 \mathrm{~ns}$ repetition interval) pulse train, with a corresponding rigorous periodicity in the spectral domain. To permit the coverage of an entire optical octave, the bandwidth of the comb emitted from the laser is further broadened by launching the pulse train into a microstructure fiber [14]. The interval between adjacent frequencies in this periodically spaced "comb" is directly defined by the pulse repetition rate. The other degree of freedom is the rate of slipping of the carrier-envelope phase of these short pulses. The generation of ultrashort pulses requires that the group velocity $\left(v_{g}\right)$ dispersion inside the laser cavity is minimized across the pulse's frequency spectrum. This criterion is not directly related to the frequency comb spacing, since the individual mode frequencies correspond 


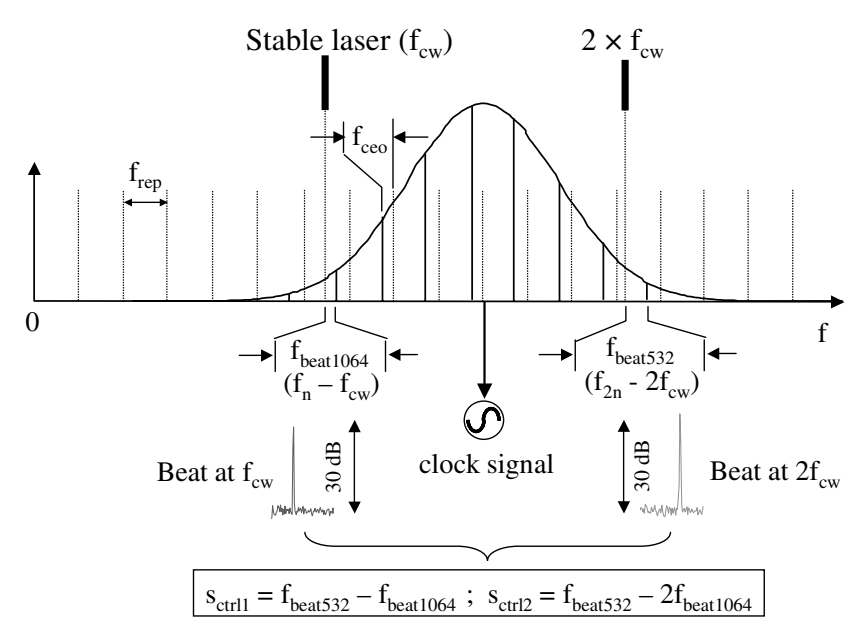

FIG. 1. The schematics of the octave-bandwidth optical comb and the stabilized $\mathrm{cw}$ laser used for control of the comb. The beat notes of the cw laser $\left(f_{\mathrm{cw}}\right)$ and its second harmonic $\left(2 f_{\mathrm{cw}}\right)$ against the corresponding comb components located, respectively, at the two comb ends are shown. The two beat signals serve as the two observed variables that are used to derive the orthogonal control signals for the comb.

to eigenmodes of the phase velocity $\left(v_{p}\right)$ of the light, and in general we have $v_{g} \neq v_{p}$. With these considerations, each optical comb frequency is therefore effectively given by $f_{n}=n f_{\text {rep }}+f_{\text {ceo. }}$. Here $n$ represents the integer $\left(\sim 3 \times 10^{6}\right)$ harmonic number of the optical comb line relative to the repetition rate, $f_{\text {rep }}$, and $f_{\text {ceo }}$ (also called the carrier-envelope offset frequency) is the comb offset frequency from the exact harmonics of $f_{\text {rep }}$ [15].

The two variables of the comb can be expressed in the following: $f_{\text {rep }}=v_{g} / l_{c}$ and $f_{\text {ceo }}=\nu_{0}\left(1-v_{g} / v_{p}\right)$, where $\nu_{0}$ is the laser carrier frequency and $l_{c}$ is the cavity length. If one is interested only in a stable $f_{\text {rep }}$, for example, to generate a clock signal in microwave, then control of $l_{c}$ seems to be a natural choice and is sufficient. Only when the entire comb spectrum needs to be stabilized then we need another control freedom, either the pump laser power which influences both $v_{g}$ and $v_{p}$, or a swivel mirror reflecting the dispersed spectrum inside the laser cavity to control $v_{g}$ [1]. Therefore some degree of signal mixing/feedforward is needed. We would use a fast servo loop acting on $l_{c}$ to stabilize $f_{\text {rep }}$, with use of the second control loop to influence mainly $f_{\text {ceo. }}$. The inevitable variation in $v_{g}$ caused by the second loop is then compensated by a properly scaled and opposite-sign feedforward signal to the first loop.

The remaining question is how to have two experimental observables to recover the information relating to $f_{\text {rep }}$ and $f_{\text {ceo. }}$. In our case we use two beat signals between a cw stabilized laser and its second harmonic against two respective comb components near the two ends of the comb spectrum. The $\mathrm{cw}$ reference laser is a $\mathrm{Nd}$ :YAG laser (frequency $f_{\mathrm{cw}}$ ) with its second harmonic $\left(2 f_{\mathrm{cw}}\right)$ locked on an iodine transition near $532 \mathrm{~nm}$ : this system offers the stability of $5 \times 10^{-14}$ at $1 \mathrm{~s}$ [16].
Both beat signals, $f_{\text {beat } 1064}=n \times f_{\text {rep }}+f_{\text {ceo }}-f_{\text {cw }}$ and $f_{\text {beat } 532}=2 n \times f_{\text {rep }}+f_{\text {ceo }}-2 f_{\text {cw }}$, are recovered with about $30 \mathrm{~dB}$ signal-to-noise ratio in a $100 \mathrm{kHz}$ bandwidth, as shown in Fig. 1. These beat signals are regenerated electronically using the rf trackingoscillator/filter approach and are then mixed in the following way to produce control signals related to $f_{\text {rep }}$ and $f_{\text {ceo }}$ : namely, $\quad s_{\text {ctrl1 }}=f_{\text {beat } 532}-f_{\text {beat } 1064}=n \times f_{\text {rep }}-f_{\text {cw }}$ and $s_{\text {ctrl2 }}=f_{\text {beat } 532}-2 f_{\text {beat } 1064}=-f_{\text {ceo. }}$. Therefore the frequency/phase variations arising in both $f_{\text {rep }}$ and $f_{\text {ceo }}$ are now directly manifested in the two control variables $s_{\mathrm{ctrl} 1}$ and $s_{\mathrm{ctrl} 2}$ and are linked to the optical frequency standard $f_{\mathrm{cw}}$. These two signals can then drive the two control transducers mentioned above to close the servo loops.

To demonstrate the effectiveness of our orthogonalized control scheme, we first show the stabilization of $f_{\text {rep }}$ to the optical standard. Essentially we need to use only the information of $s_{\mathrm{ctrl} 1}$ to control $l_{c}$ and thus $f_{\text {rep. }}$. This approach magnifies the noise of $f_{\text {rep }}$ relative to the optical standard by a factor $\sim 3 \times 10^{6}$. In doing so, we can leave the variable $f_{\text {ceo }}$ free-running since it has been effectively taken out of the control equation. In practice we use $l_{c}$ to control the phase of $s_{\text {ctrl } 1}$ to that of another stable oscillator in the rf domain (which basically translates the frequency of the optical standard by a small offset with negligible degradation of stability). Figure 2 shows the time record of the frequency differences between $f_{\mathrm{cw}}$ and $2813988 \times f_{\text {rep }}$, with a standard deviation of $0.8 \mathrm{~Hz}$ at a 1-s counter gate time. Allan deviation calculated from this time record is shown in the bottom trace of the figure. It is satisfying to find that the tracking capability of the comb system, at a level of $10^{-15}$ or better, is more than 10 times more precise than the current optical standard itself.

With the excellent tracking property of the comb system, we expect the stability of the derived clock signal of $f_{\text {rep }}$ to be basically that of the optical standard, namely, $5 \times 10^{-14}$ at $1 \mathrm{~s}$. To characterize the system, a reality check would be to compare the optical clock signal against other well-established microwave/rf frequency standards. The international time standard, Cs clock, should certainly be one of the references. However, the short term stability of a commercial Cs atomic clock is only about $5 \times 10^{-12}$ at $1 \mathrm{~s}$. For improved short term characterization of the fs comb clock, we also use a hydrogen maser signal transmitted over a 2-km fiber, and another in-house highly stable crystal oscillator (short term stability better than $5 \times 10^{-13}$ at $1 \mathrm{~s}$ ), which is slowly slaved to the Cs reference for correcting the frequency offset and drift [17]. Figure 3 summarizes the comparison results of the optical clock against all three rf references. The upper graph shows part of the time record of the beat signal between an $8 \mathrm{GHz}$ synthesized frequency from the crystal oscillator against the 80th harmonic of $f_{\text {rep }}(\sim 100 \mathrm{MHz})$. We use the combination of high harmonic orders and 

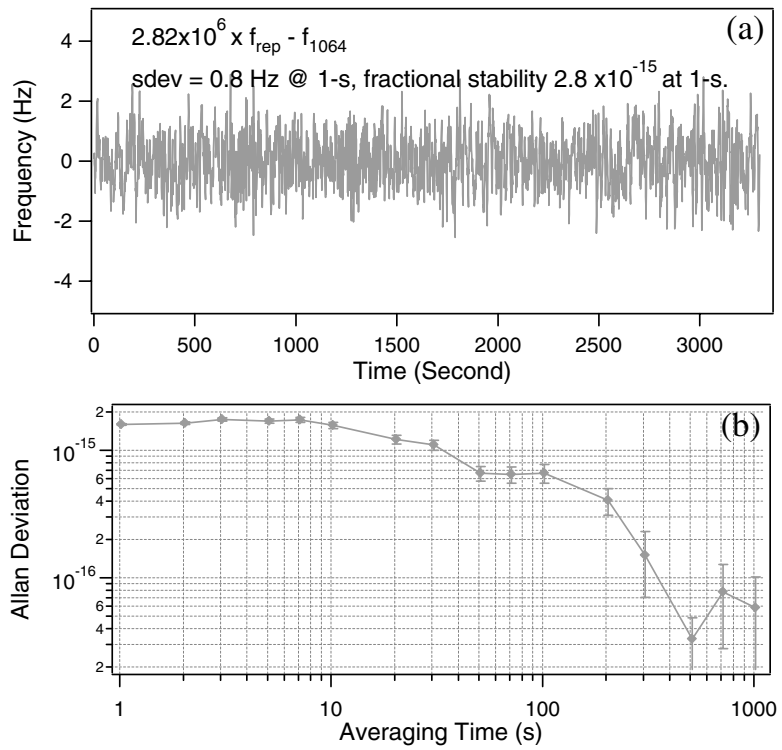

FIG. 2. Tracking stability of the comb repetition frequency to the $\mathrm{cw}$ reference laser. (a) Time record of the frequency difference between the $\mathrm{cw}$ reference laser and the $\sim 2.82 \times 10^{6}$ harmonic of $f_{\text {rep }}$. (b) The associated Allan deviation calculated from the time record.

heterodyne beat to magnify the frequency noise while making measurements at a low carrier frequency. The standard deviation of the beat frequency at 1-s averaging time is $0.0033 \mathrm{~Hz}$. The resultant Allan deviation is shown as the curve in triangles in the bottom graph of the figure. Use of a more stable hydrogen maser signal further reduces the Allan deviation of the beat, to be just below $3 \times 10^{-13}$ at $1 \mathrm{~s}$ (shown with open circles). The beat between the optical and the Cs clock is represented by the curve in diamonds. For comparison, we also display the Allan deviation associated with the Cs atomic clock ("worst case" specification) in circles and the Allan deviation of the iodine stabilized laser in squares. The data of the optical standard itself were obtained from heterodyne experiments between two similar laser stabilization systems. We note that the superior stability of our optical clock is currently not yet revealed by the microwave-clock based tests. A microwave source with a better short term stability can be substantially more expensive, even more than our optical system. Use of two optical clock systems would be the ultimate choice to perform thorough cross-checks of these optical clocks.

So far we have made an optical comb that has a welldefined frequency spacing but the absolute frequencies are uncertain since $f_{\text {ceo }}$ is left floating. An attractive approach to stabilize the entire comb spectrum is to transfer the stability of a single optical standard to the whole set of the comb components throughout the bandwidth. To accomplish this task, we need the information carried by $s_{\text {ctrl2 }}$ to exert servo action on the comb by the second control parameter, in our case, the swivel mirror. When this second loop is activated, the impact to the first loop where $f_{\text {rep }}$ is
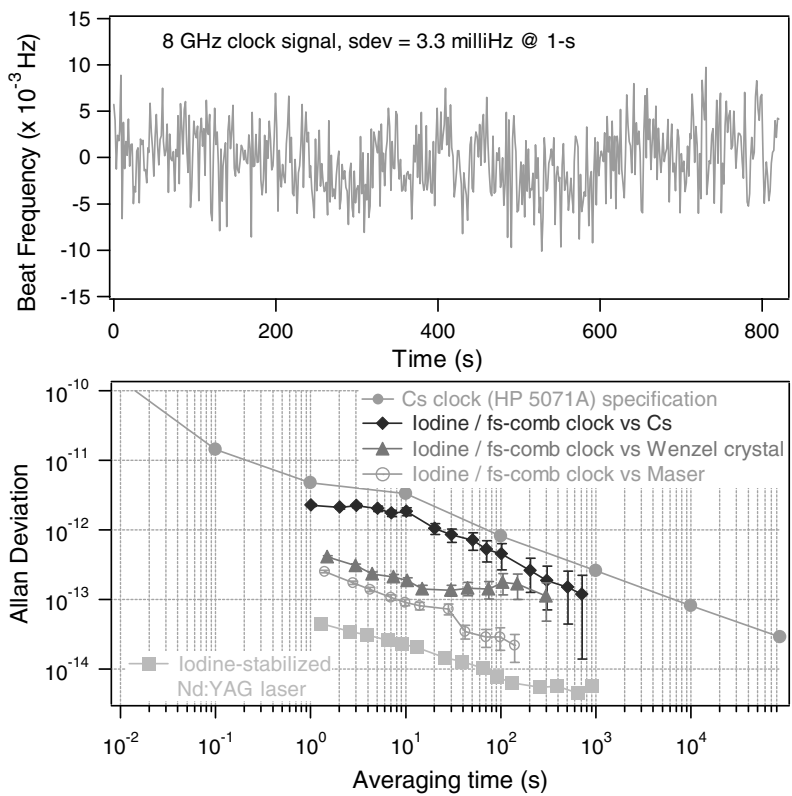

FIG. 3. Characterization of the clock signal derived from the iodine stabilized laser. The upper graph shows part of the time record of the beat frequency between an $8 \mathrm{GHz}$ synthesized frequency of the crystal oscillator referenced to a Cs clock against the 80th harmonic of $f_{\text {rep }}$ of the comb. The lower graph shows the relevant Allan deviations: squares for iodine stabilized laser; circles for the upper stability limit of the Cs atomic clock; triangles, open circles, and diamonds for the beat between the optical clock and the crystal oscillator, the maser, and the Cs clock, respectively.

being stabilized through $l_{c}$ is small. This is partly due to the fact that the dependence of $f_{\text {rep }}$ and $f_{\text {ceo }}$ on their respective control variables is to a large degree already well separated. The other part of the reason is that fluctuations of $f_{\text {ceo }}$ develop on a slower time scale compared with that of $f_{\text {rep }}$ and therefore a correspondingly slower servo loop is sufficient for stabilization of $f_{\text {ceo }}$.

We use the two original optical beats, namely, $f_{\text {beat } 1064}$ and $f_{\text {beat532 }}$ that are responsible for generating the control observables but are otherwise outside the servo loops, to characterize the performance of the orthogonal control of the comb. Figure 4 shows the counting record of the two beat frequencies of $f_{\text {beat } 1064}$ and $f_{\text {beat } 532 \text {. Both signals are }}$ shown with their mean values removed but indicated in the figure. Again the counter gate time is $1 \mathrm{~s}$ and the standard deviations of the two beat signals are $1.7 \mathrm{~Hz}$ for $f_{\text {beat532 }}$

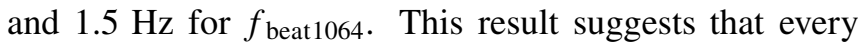
comb component over the entire optical octave bandwidth is following the $\mathrm{cw}$ laser standard at a level of $3.5 \times 10^{-15}$, again a factor of roughly 10 times better than the current optical standard itself. The future implication of this work is very clear: with an appropriately chosen optical standard, we can establish an optical frequency grid with lines repeating every $100 \mathrm{MHz}$ over an octave bandwidth and with every line stable at the $1-\mathrm{Hz}$ level.

The long term stability/reproducibility of the iodine stabilized laser is characterized by comparison against the 

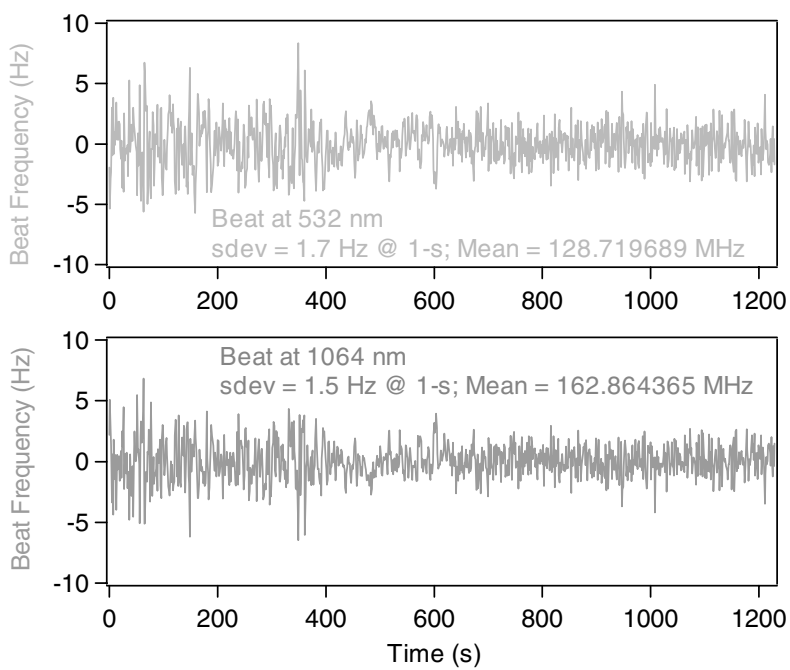

FIG. 4. Orthogonal control of the entire optical comb, showing $\mathrm{Hz}$-level stability for both beat signals of the $\mathrm{cw}$ laser against a comb component at $1064 \mathrm{~nm}$ (bottom trace) and the second harmonic of the $\mathrm{cw}$ laser and its corresponding comb line at $532 \mathrm{~nm}$ (upper trace). Better orthogonalization in the control loops leads to reduced noise after $400 \mathrm{~s}$.

Cs clock over a period of more than one year. In this long term comparison, basically we measured repeatedly the stabilized laser frequency using the Cs-referenced optical comb. During the measurement period, we changed a number of parameters associated with the comb and its generation, including $f_{\text {rep }}$, laser power, spectrum and pulse width, and the nonlinear fiber lengths, etc. The reference $\mathrm{Nd}$ :YAG laser is stabilized on the $\mathrm{R}(56) 32-0 a_{10}$ iodine transition via a modulation transfer technique. The iodine cell is $1.2 \mathrm{~m}$ long and its vapor pressure maintained by its cold finger $\left(-15^{\circ} \mathrm{C}\right)$ is $0.787 \mathrm{~Pa}$. The pump (probe) laser power is $\sim 1.0(0.25) \mathrm{mW}$ with collimated beam diameters of $\sim 1.9 \mathrm{~mm}$. The result of this rf-optical frequency intercomparison is shown in Fig. 5 and is consistent with our previous measurement [2]. However, we are now able to show that the measurement uncertainty over the entire year is about $126 \mathrm{~Hz}$, or about $4 \times 10^{-13}$. Long term drift is not statistically significant. During the last month of the data record, after the optical comb system was further improved, the standard deviation was reduced to $16 \mathrm{~Hz}$ $\left(6 \times 10^{-14}\right)$. This result represents the lowest level of uncertainty associated with any compact, cell-based optical frequency standards.

A portable version of this iodine based optical clock would have a great impact in field applications: we will be able to make precision measurements in length and time with a single device. The frequency stability in both microwave and optical domains is exceptional, surpassing basically all common sources save only national-standardsscale massive installations. The stability $\left(5 \times 10^{-14}\right.$ at $1 \mathrm{~s})$ and reproducibility $\left(4 \times 10^{-13}\right)$ of the cw laser locked

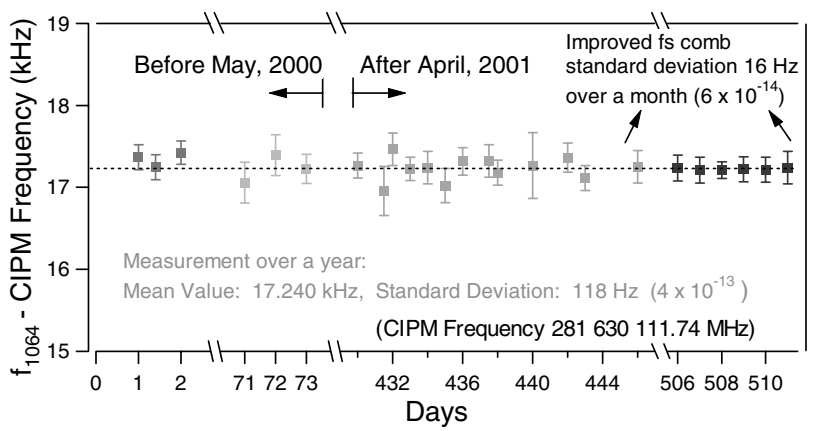

FIG. 5. Long term reproducibility of the intercomparison between the Cs clock and the iodine stabilized laser. The measurement period covers more than one year with the stability level at the $4 \times 10^{-13}$. This represents the upper limit of the long term reproducibility of our current optical standard as well as the reproducibility of the comb-based frequency transfer mechanism. CIPM: The Comité International des Poids et Mesures.

on the iodine transition can be further improved, possibly by another factor of 10 . We fully expect such simple optical clock systems will be developed by various interested laboratories.

We thank J. L. Peng, T. Fortier, R. Shelton, S. Cundiff, S. Diddams, L. Hollberg, J. Bergquist, H. Kapteyn, John Kitchin, and J. Levine for useful discussions. The work at JILA is supported by NASA, NIST, NSF, and the Research Corporation. J. Y. and J.L. H. are staff members of NIST Quantum Physics Division.

*Corresponding author.

Email address: ye@jila.colorado.edu

[1] Th. Udem et al., Phys. Rev. Lett. 82, 3568 (1999); Th. Udem et al., Opt. Lett. 24, 881 (1999).

[2] S. A. Diddams et al., Phys. Rev. Lett. 84, 5102 (2000).

[3] M. Niering et al., Phys. Rev. Lett. 84, 5496 (2000).

[4] J. Ye et al., Phys. Rev. Lett. 85, 3797 (2000).

[5] Th. Udem et al., Phys. Rev. Lett. 86, 4996 (2001).

[6] D. J. Jones et al., Science 288, 635 (2000).

[7] S. A. Diddams et al., Science 293, 825 (2001).

[8] L.-S. Ma et al., Phys. Rev. A 64, 021802(R) (2001).

[9] R. K. Shelton et al., Science 293, 1286 (2001).

[10] L. Hollberg et al., IEEE J. Quantum Electron. (to be published).

[11] R. J. Rafac et al., Phys. Rev. Lett. 85, 2462 (2000).

[12] J. Ye et al., Opt. Lett. 25, 1675 (2000).

[13] M. T. Asaki et al., Opt. Lett. 18, 977 (1993).

[14] J. Ranka et al., Opt. Lett. 25, 25 (2000).

[15] J. Reichert et al., Opt. Commun. 172, 59 (1999).

[16] J. Ye et al., IEEE Trans. Instrum. Meas. 48, 544 (1999); J. L. Hall et al., ibid. 48, 583 (1999).

[17] Cs clock: HP 5071 A; crystal oscillator: Wenzel 5/10 Blue Top Ultra Low Noise Oscillator; hydrogen maser: ST-22, Clock-13, NIST Boulder. Mentioning of commercial products is for technical communications only. 